\title{
Routing Optimization in IP Networks Utilizing Additive and Concave Link Metrics
}

\author{
Anton Riedl, Member, IEEE, and Dominic A. Schupke, Member, IEEE
}

\begin{abstract}
Routing optimization provides network operators with a powerful method for traffic engineering. Its general objective is to distribute traffic flows evenly across available network resources in order to avoid network congestion and quality of service degradation.

In this paper we consider routing optimization based on conventional routing protocols where packets are forwarded hop-by-hop in a destination-based manner. Unlike other work in this area, we consider routing protocols, which are able to take into account concave routing metrics in addition to additive ones. The concave link metric introduces an additional degree of freedom for routing optimization, thus, increasing its optimization potential. We present and evaluate a mixed-integer programming model, which works on these metrics. This model unifies the optimization for single-metric and dual-metric routing concepts and also includes the consideration of multipath routing. Furthermore, we propose a heuristic algorithm usable for larger network instances.

Numerical results indicate that employment of both the dual-metric concept and multipath routing can achieve considerably better utilization results than default-configured single-metric routing. A significant finding is that metric-based routing optimization with two link metrics often comes close to the results obtainable by optimization of arbitrarily configurable routing.
\end{abstract}

Index Terms-Genetic algorithm, Internet, mixed integer programming, routing optimization, traffic engineering.

\section{INTRODUCTION}

$\mathbf{T}$ RAFFIC engineering has become an important issue in Internet Protocol (IP) based networks, which have to cope with growing traffic loads as well as increasingly strict quality of service (QoS) requirements [1]. Even in well-dimensioned networks, temporary demand variations and traffic fluctuations can create overload at individual links. In order to avoid potential QoS degradation, it is therefore necessary to monitor the state of a network and to intervene whenever link utilization values approach a certain level. Routing optimization, as a method of traffic engineering, provides a means to alleviate QoS problems caused by skewed traffic loads. It is applicable in networks, which experience localized traffic congestion while still having free bandwidth resources in other areas. By adjusting the routing pattern it might be possible to shift traffic from crowded links

Manuscript received August 10, 2004; revised February 2, 2006; approved by IEEE/ACM TRANSACTIONS ON NETWORKING Editor T. Griffin. This paper is part of work done at the Institute of Communication Networks at Technische Universität München, Munich, Germany.

A. Riedl is with the Department of Physics, Computer Science and Engineering, Christopher Newport University, Newport News, VA 23606 USA (e-mail: Anton.Riedl@ieee.org; riedl@pcs.cnu.edu).

D. A. Schupke is with Nokia Siemens Networks GmbH \& Co. KG, 81730 München, Germany (e-mail: dominic.schupke@nsn.com).

Digital Object Identifier 10.1109/TNET.2007.902546 to lightly utilized network regions, thus, avoiding overload and keeping up the desired QoS.

The routing optimization approach considered in this paper is based on native IP routing where packets are forwarded in a next-hop destination-based manner along paths that were determined by the routing protocol. When computing the paths, routers take into account specific metric values associated with every link. While these link metrics usually have a physically relevant meaning such as propagation delay, cost, or bandwidth, they can also be used in a generic way purely for the sake of routing optimization. By modifying the link metric values, the path pattern of traffic flows through the network can be manipulated. Since routers exchange link information and recalculate routes automatically, this form of traffic engineering requires only little administrative effort. After changing link weights, routers adjust the paths autonomously while no special action has to be taken by the administrator. However, this simplicity also has its drawback. Due to possible temporary inconsistencies during rerouting processes, packets might be dropped or delayed, causing service quality to degrade. Therefore, this optimization method is mainly applicable for medium and long-term adjustments.

By default, most conventional routing protocols base their path computation only on one additive link metric, which typically results in shortest-path routing. However, some protocols allow more than one type of metric being taken into account when calculating the forwarding paths. In this paper, we specifically focus on routing protocols that support additive as well as concave link metric types. As we will discuss later, an additional concave link metric introduces more routing flexibility and, thus, offers greater optimization potential. We will show that results obtained for two link metric types often surpass the results achieved for a single additive metric. This suggests that operators should take advantage of the second link metric where possible. Furthermore, the findings of this paper can be an incentive for including more link metric types in protocols, which only support an additive metric so far.

The remainder of the paper is organized as follows. In Section II, we describe route computation issues for multimetric routing protocols as these provide the basis for our routing optimization approach. In Section III, an overview of related work on routing optimization is given. In Section IV, we formulate and discuss a generic mixed-integer optimization model, which considers path computation with additive and concave link metrics. We propose and evaluate a heuristic genetic algorithm in Section V. In Section VI, we present numerical results obtained for several scenarios with single and dual-metric routing, allowing single as well as multiple paths. Furthermore, an applicability assessment of the formal optimization approach is included. We summarize and conclude in Section VII. 


\section{ROUTING OPTIMIZATION FUNDAMENTALS}

In this section we describe the underlying principles of our optimization model. Doing so, we differentiate between singlemetric and multiple-metric (in our case, dual-metric) routing protocols. For further references, we pick one representative implementation for each category. While various protocols could be selected, $O S P F$ is chosen as the representative of singlemetric routing protocols and EIGRP is used for demonstrating the features of multiple-metric protocols.

Open Shortest Path First (OSPF) was standardized by the IETF in 1998 [2]. It allows four different types of metrics being associated with every link: distance, cost, delay, and reliability. However, for path computations only one metric is used at a time. Theoretically, it would be possible to compute four different routing tables (one for each metric type) and, then, forward packets according to one of the schemes. To guarantee consistency among all routers, this would require that IP packets are marked appropriately and that every router applies the same forwarding scheme. In practice, this feature is not used and routes are computed only for one additive metric type.

The Enhanced Interior Gateway Routing Protocol (EIGRP) is a protocol proposed by Cisco [3]. With EIGRP, every interface (i.e., link) has four different metric types associated with it: delay, bandwidth, reliability, and load, which all can be considered for path computation. The first two parameters are assigned statically, while the third and the fourth are determined by the routers during network operation. When a router computes the path towards a destination, it considers a combination of these metric parameters. The overall path metric function is given by

$$
M=\left[K_{1} \cdot b+\frac{K_{2} \cdot b}{256-\text { load }}+K_{3} \cdot d\right] \cdot \frac{K_{5}}{\text { reliability }+K_{4}}
$$

with

$$
b=256 \cdot \frac{10^{7}}{\min (\text { bandwidth })}
$$

and

$$
d=256 \cdot \text { delay }
$$

The individual components of the metric sum can be interpreted as follows.

- The first term concerns the bottleneck bandwidth $(\min ($ bandwidth $))$, i.e., the smallest link capacity along the path, which is given in kbps. The smaller the bottleneck bandwidth is, the more it contributes to the overall path metric sum. As a consequence, it is less likely that a path with a small bottleneck bandwidth is selected for routing.

- In the second term, a load parameter is introduced, which represents the utilization of the highest loaded link along the path. This parameter enables adaptive routing where routes are adjusted to current load situations and links with very high loads are avoided. However, it is well known that this kind of routing adaptation might lead to unpredictable and rather undesirable effects such as route flapping and

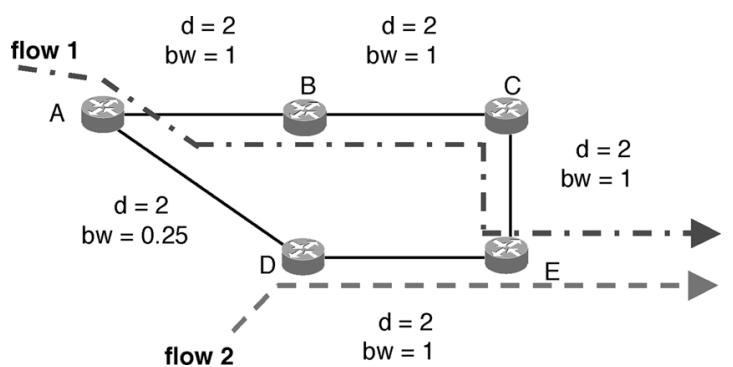

Fig. 1. Routing with additive and concave link metrics.

corresponding routing instability [4]. Therefore, it is recommended that this option be deactivated by setting the weight factor $K_{2}$ to 0 .

- The third item of the sum accounts for the total propagation delay, which is the sum of all individual link delays along the path. The link delay values are configured statically by the network administrator. It is noteworthy that the delay parameter is the only additive component of the path metric.

- The last term stresses the issue of reliability. The parameter reliability corresponds to reliability of the least-reliable link along the path. It is a probabilistic measure, which routers observe during run-time. The higher the reliability of a path is, the more preferable it appears for a router. With the two weight parameters $K_{4}$ and $K_{5}$ the influence of this metric can be adjusted. By definition, setting $K_{5}=0$ turns off the reliability option.

In default router configurations, the weight factors are $K_{1}=$ $K_{3}=1$ and $K_{2}=K_{4}=K_{5}=0$, which leaves us with the simplified path metric formula

$$
M_{\text {default }}=\left[\frac{10^{7}}{\min (\text { bandwidth })}+\text { delay }\right] \cdot 256 .
$$

This setting only activates two metrics, one additive metric (delay) and one concave metric (bandwidth). Taking the default configuration, routing preference is given to paths that are shortest in terms of a combination of low total delay and high throughput. Without loss of generality, we normalize the metric function $M_{\text {default }}$ and use following expression instead:

$$
\begin{aligned}
M_{\text {norm }} & =\left[K_{1} \cdot \frac{1}{\min _{l}\left(b w_{l}\right)}+K_{3} \cdot \sum_{l} d_{l}\right] \\
& =\left[K_{1} \cdot \max _{l}\left(i c m_{l}\right)+K_{3} \cdot \sum_{l} d_{l}\right]
\end{aligned}
$$

where $l$ are the links along a path. Parameters $b w_{l}$ and $d_{l}$ are the properly scaled bandwidth and delay values of the links. Parameter $i c m_{l}=1 / b w_{l}$ denotes the inverse capacity metric, which we use instead of the bandwidth. To illustrate the impact of bandwidth-related metric components see Fig. 1. Link A-D has only a (normalized) capacity of 0.25 and, therefore, contributes to $M_{\text {norm }}$ with a reciprocal bandwidth metric of $1 / 0.25=4$. Thus, the cost of path A-D-E is 8 , which is the sum of the delay values plus the bandwidth component. As a consequence, router A chooses router B as its next-hop neighbor, 


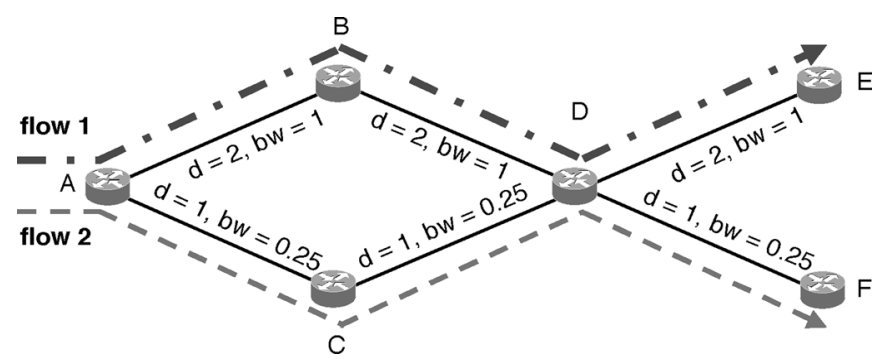

Fig. 2. Fish pattern routing with EIGRP.

where the metric total is only 7 . Note that the choice of outgoing interface for router $\mathrm{A}$ would be $\mathrm{D}$ if only the additive delay values $d$ were considered.

Depending on the scaling of values $d$ and $i \mathrm{~cm}$, emphasis is put either on small overall delay or on high throughput. In the case that delay metrics are substantially larger than inverse capacity metrics, the overall path metric is mainly determined by delay values. Only when there are several path alternatives with equal smallest delay sum does the bandwidth component really matter. The router then selects an outgoing interface with the largest possible throughput (widest-shortest path). In the opposite case $(i \mathrm{~cm} \gg d)$, high throughput paths are preferred, and delay is mainly used to break ties (shortest-widest path). Whenever the two link metrics are of the same order, no clear preference is given to either one of them. Link metrics are then used in their most generic form as a means of routing optimization, without any physically relevant meaning.

Routing based on multiple metrics provides greater optimization potential than its single-metric counterpart. It can realize additional routing patterns as it allows finer granularity of flow treatment in networks with alternative paths. This can be demonstrated in the network scenario in Fig. 2. Assume we have two traffic flows with different destinations, whose paths have several nodes in common. Let A be the first node where the two flows come together and $\mathrm{D}$ be the last common node on their way. While single-metric shortest-path routing would merge the flows at node A and send both of them either over B or over C, multiple-metric routing protocols can achieve the flow pattern shown in the figure. In the given example, the chosen path for flow 1 has a total metric sum of 7 , while the link metric values along the route via $\mathrm{C}$ would sum up to 8 . For flow 2 the situation is different. The total metric sums of the path over B and the path over $\mathrm{C}$ are 9 and 7 , respectively. The trick is to use the inverse capacity metric to make one path option appear more costly for one traffic flow, while for the other flow a larger $i \mathrm{~cm}$ value has no extra effect. It experiences already high $i \mathrm{~cm}$ values on other links along the path, which the two flows do not share. Note that it is always possible for dual-metric routing to produce a solution of single-metric protocols. Setting $i \mathrm{~cm}=1$ for all links practically corresponds to single-metric routing.

\section{A. Multi-Path Routing}

Both protocols, OSPF and EIGRP, support multiple paths towards one destination. OSPF allows equal-cost load sharing, which means that traffic for a certain destination is split equally over all routes with the same shortest path lengths (equal-cost

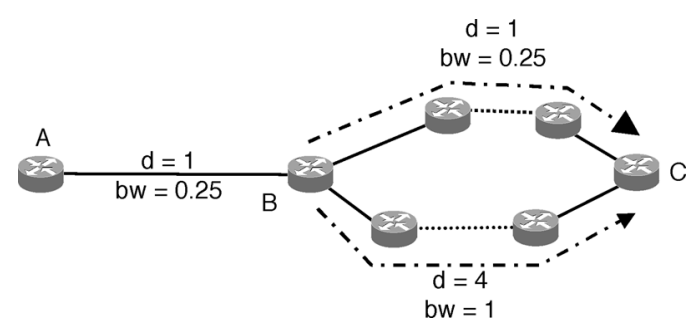

Fig. 3. ECMP routing.

multipath, ECMP). EIGRP supports multipath routing even over paths, which do not have exactly the same metric sum. However, due to the inherent complexity of this option we do not consider it in our optimization.

In the case of multiple-metric routing protocols, "equal cost" does not necessarily mean that all metric components are the same on all load-sharing paths. This is illustrated in Fig. 3. While the final weight combinations of both paths from $\mathrm{B}$ to $\mathrm{C}$ are equal, their delay and bandwidth portions are quite different. In order to compute the route from $\mathrm{A}$ to $\mathrm{C}$, router $\mathrm{A}$ needs to know metrics $d$ and $i \mathrm{~cm}$ of the path from B to C. Therefore, B has to choose one representative interface among the outgoing interfaces, whose metric set is then passed on to the upstream routers. Although both paths have equal costs, the choice of metric pair that B reports to A affects the cost value of A towards C. Receiving the metric values of the upper path results in a total cost of 6 , while the lower path metric sum would give a value of 9. To allow unambiguous route computation we define a primary outgoing interface, whose metric values are reported to neighbor routers. In this work, we assume the following assignment: Among all outgoing interfaces, the primary interface is the one with the highest throughput value (i.e., lowest $i \mathrm{~cm}$ ) towards the destination. In the given example, this would be the lower path with $i \mathrm{~cm}=b w=1$.

\section{RELATED WORK}

Routing optimization in IP networks has received a considerable amount of attention in recent literature. The great majority of publications consider routing optimization based on Multiprotocol Label Switching (MPLS) [5], which allows the setup of explicit paths between two end nodes. However, as this is not the topic of our paper, we do not review relevant literature at this point. For a technical introduction of this topic, see [6].

The following publications deal with routing optimization based on native IP routing. If at all relevant, the authors assume that the underlying shortest-path algorithm uses exactly one additive link metric. We do not know of any other work where an additional concave metric would play a role for optimization issues.

In [7], Bley et al. consider routing optimization as an embedded problem of network planning for IP networks. While the overall objective is the minimization of capacity costs, the final routing pattern has to follow the typical IP next-hop destination-based paradigm. The problem is formulated as a mixed-integer program where only one additive metric is considered. One feature of the approach is that it ensures uniqueness of shortest paths. It does not allow taking into account the possibility of 
load-sharing across equal-cost paths. As the overall problem becomes very complex even for medium-size networks, heuristics are presented for finding an initial topology and for improving it. The heuristic is based on a local search, which iteratively scans the neighborhood for better solutions.

In [8] and [9], Fortz et al. motivate the idea of using OSPF/IS-IS routing with optimized weight settings in order to improve network QoS. The optimization objective is $\sum_{l \in \text { links }} f\left(\rho_{l}\right)$, where $f\left(\rho_{l}\right)$ is a piecewise linear and convex function over link utilization $\rho_{l}$. As $\rho_{l}$ approaches 1, the slope of $f$ drastically increases, causing the optimization process to avoid link overload situations if possible. For solving the optimization problem, a guided search heuristic similar to tabu search is proposed. This heuristic explores the neighborhood of an initial weight vector by modifying individual link metric settings in a greedy fashion. In the case of equal shortest paths, the load is evenly split up (OSPF ECMP). To diversify the search process, random perturbation of link weights is performed after finding a local minimum. In [10], further investigations of routing optimization are presented, taking into account failure situations, multiple load periods, as well as noisy traffic matrices.

The work of Ericsson et al. [11] considers the same optimization problem with identical objective function as in Fortz's work. However, a different search strategy for the weight setting problem is proposed. Ericsson's approach is based on a genetic algorithm, where individual solutions of the problem are represented by means of the link weights. This application of the genetic algorithm framework corresponds in principle to the one presented in [12]. Resende presents a memetic version of the algorithm, which in addition to the genetic algorithm implements a local search heuristic [13].

In [14], Ramakrishnan and Rodrigues discuss different forms of shortest-path routing optimization. In this context, they introduce the $N$ commodity linear program formulation for the general multicommodity (MC) flow problem [15]. This formulation takes into account the destination-based forwarding behavior of current intra-domain routing protocols. It requires fewer variables and, thus, is more efficient than the general MC formulation. However, the formulation is not capable of capturing all relevant characteristics of the OSPF protocol. Therefore, a combinatorial search procedure is presented. Starting from an initial network configuration, the link weights are iteratively modified in order to drive network QoS towards a local optimum. The average network delay (as computed for $\mathrm{M} / \mathrm{M} / 1$ waiting systems) serves as an objective.

Wang et al. [16] also approach the routing optimization problem through use of the MC flow formulation. Their primary objective is the minimization of the maximum link utilization, which results in a well-balanced network. They show that any set of optimal routes which is obtained from the MC linear program can be represented by shortest path routing where the link weights are determined by the dual problem. However, the presented approach does not capture realistic features of existing protocols such as the need for integer link weights or the incapability of arbitrarily distributing traffic across equal-cost paths.

In [17], Sridharan et al. address the constraint that a router is only able to distribute traffic evenly across all shortest-path next hops. It is assumed that routing optimization is done in a transport network, where a destination node corresponds to an egress router, which represents several network prefixes, i.e., flow destinations outside the considered autonomous system. In this case, traffic flows towards a certain destination node can be differentiated according to their routing prefixes and routing optimization can then be performed on basis of these sub-flows. Since this allows finer-granular treatment of traffic flows, the potential of shortest-path routing optimization is greatly increased. The authors present several algorithms to compute the link weights in the network as well as the assignments of sub-flows to appropriate next hop interfaces for each router. A great drawback of this scheme is that it requires network administrators to explicitly modify the routing tables within the routers. After routers have autonomously calculated the routing tables, one has to change table entries associated with certain prefixes in order to obtain the desired routing pattern.

Further work dealing with routing optimization in the context of traffic engineering and performance improvement has been published by Staehle et al. [18], [19]. The optimization problem is formulated as a mixed-integer program, which is then solved with CPLEX [20]. As objective function, a weighted sum of the average and the maximum link utilization is considered. Due to the complexity of the problems, which increase with the number of nodes, links, and flows, the approach only works for smaller network sizes. For a larger network, the solver could not always find the optimum solution. Heuristic approaches are not presented by the authors. However, a separation algorithm is proposed, which breaks down large networks into smaller areas. These are then dealt with individually before being reunited [21].

In [22] and [23], Mulyana and Killat propose a hybrid genetic algorithm for the routing optimization problems. The objective function considers a weighted sum of the average and the maximum link utilization in the network. In another approach, the maximum link utilization is replaced by the number of modified link weights. This way, weight changes can be taken into account and minimized.

More work about routing optimization in the context of network dimensioning is presented by Holmberg and Yuan [24], [25]. This work extends the mathematical formulation of [7] and introduces the possibility of equal-cost load-sharing paths. The objective function is again the total sum of all cost values in the network plus a penalty component for traffic demand, which cannot be served. The problem is solved by means of a simulated annealing algorithm, which is applied to the set of integer link weights.

\section{Mixed-Integer Programming Model}

In this section, we present the mixed-integer programming model for routing optimization. Based on a given network topology with fixed link capacities and a known demand matrix, our traffic engineering model minimizes the maximum link utilization in the network by adjusting the individual delay and inverse capacity metrics. Since these values are originally related to physical link characteristics (propagation delay or link length and capacities) and their liberal use solely for the purpose of traffic engineering might create discomfort, it should be possible to limit the range of adjustment in order to stay as close to the "physically genuine" values as desired. However, 
TABLE I

PARAMETERS OF MiXed-INTEGER PROGRAM FOR OSPF/EIGRP ROUTING OPTIMIZATION

\begin{tabular}{|l|l|}
\hline node set & $\mathcal{V}$ \\
\hline edge set & $\mathcal{E}$ \\
\hline link capacities & $C_{i j}$ \\
\hline link delay metric limits & $D_{i j}^{\text {min }}, D_{i j}^{\text {max }}$ \\
\hline inverse capacity metric limits & $I C M_{i j}^{\text {min }}, I C M_{i j}^{\text {max }}$ \\
\hline traffic demand & $A[u, v], \hat{A}[v]$ \\
\hline set of distinct $i c m$ values & $\left\{\widetilde{I C M}_{1}, \ldots, \widehat{I C M} \widetilde{M}\right\}$ \\
\hline incremental $i \mathrm{~cm}$ vector & $\mathbf{m}$ \\
\hline
\end{tabular}

one must be aware that this restriction might also limit the traffic engineering potential.

During our traffic engineering procedure, we allow link utilization values to be greater than one. However, if after the optimization the maximum utilization value is still greater than one (or greater than any desired threshold), the network has to be regarded as being overloaded and capacities need to be extended. The value of the threshold usually depends on the type of traffic, which is carried in the network. Delivering real-time traffic with appropriate quality of service requires much smaller link utilizations than transporting best-effort data.

The optimization task is stated as a mixed-integer linear program. We try to follow the routing process and the routers' computational procedure as closely as possible. We believe that this makes it easier to further extend the model in order to consider additional relevant routing features. Specifically, our model considers:

- destination-based forwarding;

- single as well as dual-metric routing protocols; and

- multipath forwarding over equal-cost paths.

\section{A. Parameters}

Table I summarizes the parameters that are needed to model the optimization problem. The IP network is modeled as a directed graph $\mathcal{G}=\{\mathcal{V}, \mathcal{E}\}$ with node set $\mathcal{V}$ and edge set $\mathcal{E}$. We refer to the nodes and the links by means of their indices, i.e., node $v$ denotes the node with index $v, 1 \leq v \leq|\mathcal{V}|$ and edge $e$ denotes the edge with index $e, 1 \leq e \leq|\mathcal{E}|$. Furthermore, an edge is also represented through its end points, i.e., edge $(i, j)$ is the edge with source node $i$ and target node $j$. Every edge $(i, j) \in \mathcal{E}$ has a link capacity $C_{i j}$ associated with it. As mentioned above, it should be possible to limit the range of delay and inverse capacity metrics for each link. Thus, parameters $D_{i j}^{\min }(\geq 1), D_{i j}^{\max }\left(\geq D_{i j}^{\min }\right), I C M_{i j}^{\min }$, and $I C M_{i j}^{\max }$ specify the minima and maxima of the delay and $i \mathrm{~cm}$ metrics for link $(i, j)$, respectively.

The traffic demand is represented by a matrix $A$ where $A[u, v]$ is the flow with origin node $u$ and destination node $v$. We assume that $A[u, v]=0$ if $u=v$. Let $\hat{A}[v]$ be the total traffic destined to node $v$, i.e., $\hat{A}[v]=\sum_{u \in \mathcal{V}} A[u, v]$.

\section{B. Optimization Variables}

Routing optimization is carried out by setting the delay and inverse capacity metric values. For every link $(i, j)$ we introduce a delay variable $d_{i j}\left(D_{i j}^{\min } \leq d_{i j} \leq D_{i j}^{\max }\right)$ and an inverse capacity metric $i c m_{i j}\left(I C M_{i j}^{\min } \leq i c m_{i j} \leq I C M_{i j}^{\max }\right)$. It is important to note that the optimization potential is directly affected by the permissible value range of the variables $d_{i j}$ and $i \mathrm{~cm}_{i j}$ as well as by their adjustment granularity. In both cases, a small range or coarse granularity limits the search space and, thus, possibly reduces the optimization gain. On the other hand, allowing greater flexibility increases the complexity of the program, which might make it intractable. This is especially evident for the $i \mathrm{~cm}_{i j}$ values where every additional value option requires the introduction of a new link variable (see next paragraph). Thus, a tradeoff is necessary, which finds a balance between optimization quality and model complexity.

Variables $i c m_{i j}$ cannot be used directly in a linear program. Since the solver has to be able to derive the maximum of two $i \mathrm{~cm}$ values during the optimization process, it is necessary that they are encoded in a special way. Therefore, we specify a finite set of $\widetilde{M}$ distinct $i \mathrm{~cm}$ values $\left\{I \widetilde{C M} M_{1}, \ldots, I \widetilde{C M} \widetilde{M}_{\widetilde{M}}\right\}$, which are sorted in decreasing order, i.e., $\widetilde{I C M}_{1}>\widetilde{I C M_{2}}>\ldots>\widetilde{I C M_{\widetilde{M}}}$, and form an incremental $i c m$ vector $\mathbf{m}=\left\{m_{1}, m_{2}, \ldots, m_{\tilde{M}-1}\right\}$ with $m_{n}=\widetilde{I C M_{n}}-\widetilde{I C M_{n+1}}$ for $n=1, \ldots, \widetilde{M}-1$. Then, a link's $i c m_{i j}$ value can be represented by the metric vector $\mathbf{m}$ and a binary indicator vector $\mathbf{b}_{i j}=\left\{b_{i j, 1}, b_{i j, 2}, \ldots, b_{i j, M}\right\}$ with $b_{i j, n} \leq b_{i j, n-1}$ for $n=2, \ldots, M$ and $M=\widetilde{M}-1$. We have $i c m_{i j}=I C M_{1}-\sum_{n=1}^{M} m_{n} \cdot b_{i j, n}$ for all $(i, j) \in \mathcal{E}$. During the optimization process, the elements of vectors $\mathbf{b}_{i j}$ are used as variables instead of $i c m_{i j}$. The reason is that this kind of encoding allows the formulation of linear constraints, which specify the maximum of two $i \mathrm{~cm}$ variables (see constraints (26) and (27) in the next section).

The amount of traffic on a link $(i, j) \in \mathcal{E}$ towards a destination node $u \in \mathcal{V}$ is represented through variables $f_{i j, u}$. This approach takes into account that packets, which are going to the same destination, are treated equally by the routers. A router does not differentiate between the packets' sources. Therefore, it is not necessary to introduce a flow variable for every sourcedestination pair as it is commonly done in multicommodity flow problems. Instead, it is sufficient to have a variable only for each destination. This follows the approach presented in [14].

In order to consider route computation with delay and $i \mathrm{~cm}$ components, we introduce several weight and potential variables as well as routing specific indicator variables. Some of the variables are just functions of others and are used for the sake of readability. They can be substituted during preprocessing stages. The choice of an outgoing interface at every node $i$ towards a destination $u$ is modeled by the binary variable $t_{i j, u}$. It is equal to 1 if node $i$ uses link $(i, j)$ to forward packets to destination $u$. Note that for ECMP a router could use several outgoing interfaces to the same destination. Therefore, a node's primary outgoing interface is indicated by the binary variable $p_{i j, u}$. To determine the outgoing interfaces, a router has to perform path computations based on a combination of link metrics. We define a node $i$ 's delay weight $\delta_{i j, u}$ to be equal to the total path delay from node $i$ to node $u$, in the case node $i$ would use node $j$ as its next-hop neighbor towards $u$. Related to the delay weight, a node $i$ 's delay potential $\Delta_{i u}$ indicates the total delay to node $u$, which it announces to its neighbors. 
TABLE II

VARIABLES OF MIXED-INTEGER PROGRAM FOR OSPF/EIGRP ROUTING OPTIMIZATION

\begin{tabular}{|l|c|}
\hline link delay & $d_{i j}$ \\
\hline inverse capacity metric & $i c m_{i j}$ \\
\hline binary indicator vector for $i \mathrm{~cm}_{i j}$ & $\mathbf{b}_{i j}$ \\
\hline link flow & $f_{i j, u}$ \\
\hline outgoing interface indicator & $t_{i j, u}$ \\
\hline primary outgoing interface indicator & $p_{i j, u}$ \\
\hline node delay weight & $\delta_{i j, u}$ \\
\hline node delay potential & $\Delta_{i u}$ \\
\hline node capacity weight & $\psi_{i j, u}$ \\
\hline binary capacity weight indicator vector & $\phi_{i j, u}$ \\
\hline node capacity potential & $\Psi_{i, u}$ \\
\hline binary capacity potential indicator vector & $\mathbf{\Phi}_{i, u}$ \\
\hline combined-weight & $\omega_{i j, u}$ \\
\hline combined-potential & $\Omega_{i, u}$ \\
\hline link utilization & $\rho_{i j}$ \\
\hline maximum utilization & $\rho_{m a x}$ \\
\hline
\end{tabular}

A node's delay potential is equal to the delay weight associated with the primary outgoing interface. Similar to the delay weight, a node's capacity weight $\psi_{i j, u}$ is introduced, that contains the inverse capacity metric, which node $i$ observes along the path to node $u$ if it used node $j$ as the respective next-hop neighbor. The capacity potential $\Psi_{i, u}$ of node $i$ holds the relevant inverse capacity metric, which depends on the selected path towards destination node $u$ (again the choice of primary outgoing interface determines the value of $\Psi_{i, u}$ ). Analog to the inverse capacity metrics $i \mathrm{~cm}$, capacity weights $\psi_{i j, u}$ and capacity potentials $\Psi_{i, u}$ are represented by the metric vector $\mathbf{m}$ and binary indicator vectors $\boldsymbol{\phi}_{i j, u}$ and $\boldsymbol{\Phi}_{i, u}$, respectively. Vectors $\phi_{i j, u}$ and $\boldsymbol{\Phi}_{i, u}$ have the same structure as $\mathbf{b}_{i j}$ defined above. Thus, $\psi_{i j, u}=\widetilde{I C M_{1}}-\sum_{n=1}^{M} m_{n} \cdot \phi_{i j, u, n}$ and $\Psi_{i, u}=$ $\widetilde{I C M}_{1}-\sum_{n=1}^{M} m_{n} \cdot \Phi_{i, u, n}$. So far, the two routing metric components have only been considered in isolation. Therefore, we bring in one more weight and one more potential variable. A node's combined-weight $\omega_{i j, u}$ combines the delay weight and the capacity weight at node $i$ towards node $u$ over link $(i, j)$. The minimum of the weights $\omega_{i j, u}$ at a node $i$ towards destination node $u$ is given by the combined-potential variable $\Omega_{i, u}$. For a certain metric setting, $\Omega_{i, u}$ is the distance between node $i$ and node $u$ according to the dual-metric function.

Finally, we specify variables $\rho_{i j}$ as well as $\rho_{\max }$, which contain the utilization of the individual links and the maximum thereof, respectively. Table II gives an overview of the optimization variables.

\section{Constraints}

At first, the limits of the delay and $i \mathrm{~cm}$ metrics are specified. For the delay variables this is done with constraints (1) and (2). For $i \mathrm{~cm}$ variables it is more complex. Since $i \mathrm{~cm}$ values are encoded through $\mathbf{b}_{i j}$ vectors, we have to translate the limits of $i \mathrm{~cm}_{i j}$ into corresponding restrictions of the elements of indicator vector $\mathbf{b}_{i j}$. Note that an entry of a vector $\mathbf{b}_{i j}$ can only be 1 if all elements to the left of it are also 1 . Furthermore, with an increasing number of 1's the represented inverse capacity metric becomes smaller. Thus, for $i \mathrm{~cm}_{i j}$ to stay below an upper bound, one has to fix the $\hat{n}_{i j}$ lowest entries, i.e., variables $b_{i j, 1}=$ $b_{i j, 2}=\ldots=b_{i j, \hat{n}_{i j}}=1$, such that $\widetilde{I C M_{1}}-\sum_{n=1}^{\hat{n}_{i j}-1} m_{n} \geq$ $I C M_{i j}^{\max }$ and $I C M_{1}-\sum_{n=1}^{\hat{n}_{i j}} m_{n} \leq I C M_{i j}^{\max }$. Accordingly, for the lower bound the $\check{n}_{i j}$ highest entries have to be set to 0, i.e., $b_{i j, M-\check{n}_{i j}+1}=b_{i j, M-\check{n}_{i j}+2}=\ldots=b_{i j, M}=0$ such that $\widetilde{I C M}_{1}-\sum_{n=1}^{M-n_{i j}} m_{n} \geq I C M_{i j}^{m i n}$ and $\widetilde{I C M_{1}}-$ $\sum_{n=1}^{M-\check{n}_{i j}+1} m_{n} \leq I C M_{i j}^{m i n}$. In summary, we have constraints (3)-(5) where $\hat{n}_{i j}$ and $\check{n}_{i j}$ are set accordingly.

$$
\begin{aligned}
d_{i j} & \leq D_{i j}^{\max } \quad \forall(i, j) \in \mathcal{E} \\
d_{i j} & \geq D_{i j}^{\min } \quad \forall(i, j) \in \mathcal{E} \\
b_{i j, n} & =1 \quad \forall(i, j) \in \mathcal{E}, n=1, \ldots, \hat{n}_{i j} \\
b_{i j, n} & =0 \quad \forall(i, j) \in \mathcal{E}, n=M-\check{n}_{i j}+1 \ldots, M \\
b_{i j, n} & \leq b_{i j, n-1} \quad \forall(i, j) \in \mathcal{E}, n=2, \ldots, M
\end{aligned}
$$

Next, the traffic flow through the network is described. Constraints (6), (7), and (8) are a modified version of the multicommodity flow formulation, which states that at each node the incoming traffic is equal to the outgoing traffic. The constraints take into account the destination-based forwarding principle of Internet routing protocols and describe the conservation law of flows at every node in the network and at the destination nodes. Contrary to the regular multicommodity flow problem, flows are not differentiated respective to their origin. As soon as two flows with the same destination node end up at the same intermediate node, they are merged.

$$
\sum_{j:(i, j) \in \mathcal{E}} f_{i j, u}-\sum_{j:(j, i) \in \mathcal{E}} f_{j i, u}=A[i, u] \quad \forall i \in \mathcal{V}, \forall u \in \mathcal{V}: u \neq I
$$

$$
\begin{gathered}
\sum_{j:(j, i) \in \mathcal{E}} f_{j i, i}=\hat{A}[i] \quad \forall i \in \mathcal{V} \\
\sum_{j:(i, j) \in \mathcal{E}} f_{i j, i}=0 \quad \forall i \in \mathcal{V}
\end{gathered}
$$

The next constraints relate to the outgoing interfaces of each node and their linkage to the respective flow variables. Equations (9) state that node $i$ does not need an outgoing interface if traffic is destined for itself. For all other destinations there has to be at least one outgoing interface (10). Constraints (11) limit the number of outgoing interfaces per destination to $T_{\max }$ (in turn limiting traffic splitting).

$$
\begin{aligned}
t_{i j, i} & =0 \quad \forall(i, j) \in \mathcal{E} \\
\sum_{j:(i, j) \in \mathcal{E}} t_{i j, u} & \geq 1 \quad \forall i \in \mathcal{V}, \forall u \in \mathcal{V}: u \neq i \\
\sum_{j:(i, j) \in \mathcal{E}} t_{i j, u} & \leq T_{\max } \quad \forall i \in \mathcal{V}, \forall u \in \mathcal{V}: u \neq i
\end{aligned}
$$

Constraints (12) and (13) establish the relationship between outgoing interfaces and traffic flow indicator variables. With (12), an outgoing interface $(i, j)$ towards $u$ is "activated" whenever a flow with destination $u$ uses link $(i, j)$. Inequality (13), which is activated only when $t_{i j, u}$ is one, guarantees that every flow towards $u$ is sent out equally on all outgoing interfaces 
$(i, j)$ towards destination $u$ whenever it passes through node $i$. The constant $\kappa_{1}$ needs to be larger than $\max _{i \in \mathcal{V}} \hat{A}[i]$.

$$
\begin{aligned}
& \kappa_{1} \cdot t_{i j, u} \geq f_{i j, u} \quad \forall(i, j) \in \mathcal{E} \forall u \in \mathcal{V} \\
& t_{i j, u}+\left(f_{i k, u}-f_{i j, u}\right) / \kappa_{1} \leq 1 \\
& \quad \forall(i, j) \in \mathcal{E}, \forall(i, k) \in \mathcal{E}: k \neq j, \forall u \in \mathcal{V}
\end{aligned}
$$

The primary interface is chosen from the set of all outgoing interfaces. Therefore, whenever a link $(i, j)$ is a primary interface, it also has to be a regular outgoing interface (14). Only one outgoing interface of node $i$ can be the primary one (15).

$$
\begin{aligned}
t_{i j, u} & \geq p_{i j, u} \quad \forall(i, j) \in \mathcal{E}, \forall u \in \mathcal{V} \\
\sum_{j:(i, j) \in \mathcal{E}} p_{i j, u} & =1 \quad \forall i \in \mathcal{V}, \forall u \in \mathcal{V}: u \neq i
\end{aligned}
$$

Now we consider the delay portion of the path metric. In (16), the delay weight of node $i$ towards $u$ associated with link $(i, j)$ is given by the sum of neighbor $j$ 's weight potential and the link delay of $(i, j)$. A node's delay potential is 0 when addressing itself (17), and greater than or equal to 1 otherwise (18).

$$
\begin{aligned}
& \delta_{i j, u}=\Delta_{j, u}+d_{i j} \quad \forall(i, j) \in \mathcal{E}, \forall u \in \mathcal{V} \\
& \Delta_{i, i}=0 \quad \forall i \in \mathcal{V} \\
& \Delta_{i, j} \geq 1 \quad \forall i \in \mathcal{V}, \forall j \in \mathcal{V}: i \neq j
\end{aligned}
$$

The next two inequalities make sure that the delay weight of the primary outgoing interface is picked as the delay potential for node $i$ towards $u$. Constraints (19) and (20) reduce to $\Delta_{i, u}=$ $\delta_{i j, u}$ if $p_{i j, u}=1$. Otherwise, they have no effect if constant $\kappa_{2}$ is only large enough $\left(\kappa_{2} \geq \sum_{(i, j) \in \mathcal{E}} D_{i j}^{\max }\right)$.

$$
\begin{aligned}
p_{i j, u}+\left(\Delta_{i, u}-\delta_{i j, u}\right) / \kappa_{2} \leq 1 \\
\quad \forall(i, j) \in \mathcal{E}, \forall u \in \mathcal{V}, \kappa_{2} \text { const } \\
p_{i j, u}+\left(\delta_{i j, u}-\Delta_{i, u}\right) / \kappa_{2} \leq 1 \\
\quad \forall(i, j) \in \mathcal{E}, \forall u \in \mathcal{V}, \kappa_{2} \text { const }
\end{aligned}
$$

Constraints (21) through (25) define capacity weights and potentials, and the special form of their respective indicator variables.

$$
\begin{aligned}
\psi_{i j, u} & =\widetilde{I C M_{1}}-\sum_{n=1}^{M} m_{n} \cdot \phi_{i j, u, n} \quad \forall(i, j) \in \mathcal{E}, \forall u \in \mathcal{V} \\
\phi_{i j, u, n} & \leq \phi_{i j, u, n-1} \quad \forall(i, j) \in \mathcal{E}, \forall u \in \mathcal{V}, n=2, \ldots, M \\
\Psi_{i, u} & =\widetilde{I C M_{1}}-\sum_{n=1}^{M} m_{n} \cdot \Phi_{i, u, n} \quad \forall i \in \mathcal{V}, \forall u \in \mathcal{V} \\
\Phi_{i, u, n} & \leq \Phi_{i, u, n-1} \quad \forall i \in \mathcal{V}, \forall u \in \mathcal{V}, n=2, \ldots, M \\
\Phi_{i, i, n} & =1 \quad \forall i \in \mathcal{V}, n=1, \ldots, M
\end{aligned}
$$

The capacity weight of an outgoing interface $(i, j)$ at node $i$ towards destination $u$ is the maximum of the predecessor's capacity potential and the link's inverse capacity metric $i \mathrm{~cm}_{i j}$.
This is the same as an "AND" operation (expressed through (26) and (27)) on the individual entries of the indicator variables.

$$
\begin{aligned}
& \phi_{i j, u, n} \leq 0.5 \cdot\left(b_{i j, n}+\Phi_{j, u, n}\right) \\
& \quad \forall(i, j) \in \mathcal{E}, \forall u \in \mathcal{V}, n=1 \ldots, M \\
& \phi_{i j, u, n} \geq b_{i j, n}+\Phi_{j, u, n}-1 \\
& \quad \forall(i, j) \in \mathcal{E}, \forall u \in \mathcal{V}, n=1 \ldots, M
\end{aligned}
$$

The primary interface is the link among all outgoing interfaces, which has the lowest capacity weight on the path towards the destination. As explained in the preceding section, this is important in cases where multipath load-sharing is applied and the different paths have different $i \mathrm{~cm}$ values. Inequalities (28) force the capacity weight of the primary outgoing interface $\left(p_{i j, u}=1\right)$ to be smaller than or at most equal to the capacity weights of all other outgoing interfaces $\left(t_{i k, u}=1\right)$. The capacity potential of node $i$ towards destination $u$ is equal to the capacity weight of the primary interface ((29) and (30)).

$$
\begin{gathered}
p_{i j, u}+t_{i k, u}+\phi_{i j, u, n}-\phi_{i k, u, n} \leq 2 \\
\forall(i, j) \in \mathcal{E}, \quad \forall(i, k) \in \mathcal{E}: j \neq k, \forall u \in \mathcal{V}, n=1, \ldots, M \\
p_{i j, u}+\Phi_{i, u, n}-\phi_{i j, u, n} \leq 1 \\
\forall(i, j) \in \mathcal{E}, \forall u \in \mathcal{V}, n=1, \ldots, M \\
p_{i j, u}+\phi_{i j, u, n}-\Phi_{i, u, n} \leq 1 \\
\forall(i, j) \in \mathcal{E}, \forall u \in \mathcal{V}, n=1, \ldots, M
\end{gathered}
$$

Finally, we combine the two metric components and look at the combined-weight and combined-potential variables. First, the combined-weight is defined as the sum of a node's delay weight and capacity weight (31). If link $(i, j)$ is an outgoing interface towards $u$, its combined-weight has to be smaller than or at most equal to any other adjacency's combined-weight (32) depending on whether this adjacency is an outgoing interface itself or not. The combined-potential of a node $i$ is the minimum over all combined-weight variables at this node (33). In the case $(i, j)$ is an outgoing interface $\left(t_{i j, u}=1\right)$, the combined-potential is equal to this link's combined-weight ((34) and (35)). For all other adjacent links with $t_{i j, u}=0$, the respective combined-weight has to be larger (at least by one) than the node's combined-potential (35). The constant $\kappa_{3}$ has to be large enough, i.e., $\kappa_{3} \geq \max _{(i, j) \in \mathcal{E}} I C M_{i j}^{\max }+\sum_{(i, j) \in \mathcal{E}} D_{i j}^{\max }$.

$$
\begin{aligned}
& \omega_{i j, u}=\delta_{i j, u}+\psi_{i j, u} \quad \forall(i, j) \in \mathcal{E} \forall u \in \mathcal{V} \\
& t_{i j, u}+\left(\omega_{i j, u}+1-t_{i k, u}-\omega_{i k, u}\right) / \kappa_{3} \leq 1 \\
& \forall(i, j) \in \mathcal{E}, \forall(i, k) \in \mathcal{E}: j \neq k, \forall u \in \mathcal{V}, \kappa_{3} \text { const } \\
& \Omega_{i, u} \leq \omega_{i j, u} \quad \forall(i, j) \in \mathcal{E} \forall u \in \mathcal{V} \\
& t_{i j, u}+\left(\omega_{i j, u}-\Omega_{i, u}\right) / \kappa_{3} \leq 1 \\
& \quad \forall(i, j) \in \mathcal{E}, \forall u \in \mathcal{V}, \kappa_{3} \text { const } \\
& t_{i j, u}+\left(\omega_{i j, u}-\Omega_{i, u}\right) \geq 1 \quad \forall(i, j) \in \mathcal{E}, \forall u \in \mathcal{V}
\end{aligned}
$$

This leaves us with the specification of the link utilization values and their maximum, formulated through (36) and (37). 
Since $\rho_{\max }$ is minimized in the objective function, it will always be equal to and never greater than the maximum utilization.

$$
\begin{aligned}
\rho_{i j} & =\sum_{u \in \mathcal{V}} \frac{f_{i j, u}}{C_{i j}} \quad \forall(i, j) \in \mathcal{E} \\
\rho_{\text {max }} & \geq \rho_{i j} \quad \forall(i, j) \in \mathcal{E}
\end{aligned}
$$

\section{Objective Function}

As it is the aim of our optimization procedure to minimize the maximum utilization on any link in the network, we define following objective function:

$$
v=\min \left(\rho_{\max }\right) .
$$

\section{E. Characteristics and Extensions}

The dual-metric routing optimization problem is $\mathcal{N} \mathcal{P}$-hard since it extends the corresponding single-metric problem, which itself is $\mathcal{N} \mathcal{P}$-hard [8]. The number of variables is in the order of $|\mathcal{E}| \cdot|\mathcal{V}|^{2}$. The number of constraints is roughly $|\mathcal{E}|^{2} \cdot|\mathcal{V}|^{2}$ for relatively small $M$. A lower bound of the problem can be computed by a modified version of the multicommodity flow problem (MC), which takes into account the destination-based forwarding approach of Internet routing. Taking only constraints (6), (7), (8), and (37) results in a linear model, which can be solved efficiently. Routing optimization with OSPF and EIGRP can never achieve a maximum utilization, which would be better than the result of the linear MC problem. In most cases, the final results lie above the lower bound.

It is straightforward to have the routing optimization model take into account only additive metrics. Introducing parameter $K_{1}$ of the EIGRP metric formula and setting it to 0 can achieve this. However, in this case it would be better to eliminate all $i \mathrm{~cm}$ related variables and constraints and replace the combinedweight and combined-potential variables in (32)-(35) with their delay-specific counterparts. The OSPF optimization model is a subset of the presented model.

Furthermore, other objective functions are conceivable. If it is desired to consider a tradeoff between the total load in the network and the utilization on the most utilized link, the objective function can be extended in the following way:

$$
v_{\text {ext }}=\min \left(\rho_{\max }+w \cdot \sum_{(i, j) \in \mathcal{E}} \rho_{i j}\right) .
$$

The two individual objectives are combined in a weighted sum. With increasing $w$, more and more flows are forced to use the shortest paths. Consequently, the number of flows taking longer routes in order to decrease the maximum link utilization is reduced.

\section{Hybrid GENETIC AlgORITHM (HGA)}

The mixed-integer program presented in the previous section is rather complex and can only be solved for smaller networks. Therefore, a heuristic algorithm was developed, which can be applied to networks of larger size. It is based on the concept of

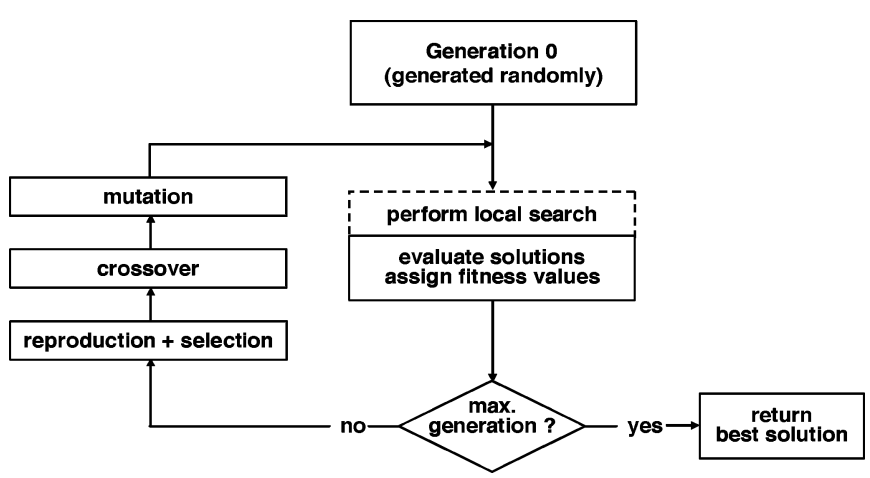

Fig. 4. Flow chart of hybrid genetic algorithm.

genetic algorithms (GAs) and its fundamental principles were published in [12]. In order to enhance the performance of the GA framework, our algorithm is complemented by a simple search heuristic [26], following the idea of [13]. In the literature, this combination is referred to as Hybrid Genetic Algorithm (HGA) or Memetic Algorithm (MA).

Genetic algorithms adopt the principles of natural selection [27], which suggests that an individual's strength to survive is determined by its gene structure. Starting with a mix of individuals and applying the basic operations of reproduction, crossover, and mutation over many generations, only strong individuals will prevail, and in fact become even stronger, whereas weak ones are reduced in number and finally die out. To apply this principle to an optimization problem, an encoding scheme is required, which allows the representation of individual solutions as strings of genes (such as binaries, integers, or characters). Furthermore, it has to be possible to quantitatively evaluate the quality of a string in order to associate a fitness value with it. This fitness value affects an individual's chance of reproduction and, thus, its likelihood of contributing to future generations. It is essential that an individual's fitness value appropriately reflects the quality of its corresponding solution.

Fig. 4 gives an overview of the algorithm structure. Starting with a randomly generated set (generation 0), the individual strings of each generation are subject to a heuristic local search algorithm, which tries to improve the quality of each string. Then the strings are evaluated according to their quality and fitness values are assigned. Next, a new generation is produced by applying the reproduction operator. Pairs of strings of the new generation are selected and crossover, i.e., the exchange of substring information, is performed. With a certain probability, genes are mutated before all solutions are again evaluated. This procedure is repeated until a maximum number of generations is reached. While doing this, the all-time best solution is stored and returned at the end.

In our implementation, we choose a string representation, which directly reflects the link metrics in the network. All links are enumerated and an integer link weight is associated with each of them. Thus, a specific gene string contains the weights of all links in the order of the links' enumeration. Based on these weights, the corresponding routing can be computed by 
applying the respective route computation algorithm. For the single-metric case, one string contains the additive metric of every link. For dual-metric routing, we require two strings for one individual, one string holding the delay values and one the $i \mathrm{~cm}$ values. The individual genes are integers ranging from 1 to a maximum value $W_{\max }$. In practice, routing protocols allow quite large maximum values (e.g., in the case of OSPF up to $65535)$. However, for traffic engineering and routing optimization purposes the metric values can be kept much smaller. In our network scenarios we assume a maximum metric value of 20 .

As we would like to minimize the maximum link utilization in the network, we choose our fitness function to be inverse proportional to this value. This way, routing solutions with smaller maximum link utilization receive higher fitness values and, thus, have a higher chance to be reproduced when setting up a new generation.

To adjust the selection process of the genetic algorithm, we apply power scaling to the fitness function:

$$
\text { fitness }=\left(\frac{1}{\rho_{\max }}\right)^{p}, \quad p>0 .
$$

With $p<1$ we can achieve that fitness values of bad solutions increase relatively to the best ones. Thus, weak solutions have a higher chance to survive, and the dominance and the reproduction speed of superior solutions is reduced. Setting $p>1$ has the adversary effect. Strong solutions are given even higher weight, while poor solutions die out faster.

For reproduction, we adopt the roulette-wheel type selection method with slot sizes that correspond to the fitness values [27]. This way, the probability that a specific string is chosen to be part of the next generation is equal to its fitness value divided by the sum of the fitness values of all individuals. To improve reproduction results, the all-time and the currently best solutions are injected into every new generation. This way, it is guaranteed that the search process keeps looking for better solutions around the so-far best solutions.

For the crossover process, pairs of strings are selected randomly and substring information is exchanged between them. The location, at which the strings are split up, is again random and might be different for every pair. In our implementation, the split-up point is uniformly distributed across the length of the strings. Finally, mutation is applied to individual genes with small probability, affecting only a few individuals of a generation.

In order to improve the performance and the speed of the genetic algorithm, the evaluation step, which returns the fitness of a solution, is preceded by a local search process. Before evaluating each routing solution, simple heuristics are used to divert traffic from the link with the highest utilization. This is done repeatedly until no further improvement can be achieved. As the search heuristic is deterministic, it is guaranteed that a certain metric string always results in the same routing solution. In the case of shortest-path routing with one additive metric, traffic can be diverted from a link by increasing its weight. Therefore, we increment the weight of the highest-utilized link and recompute the routing. If this step leads to a higher maximum utilization value in the network it is retracted and the local optimization

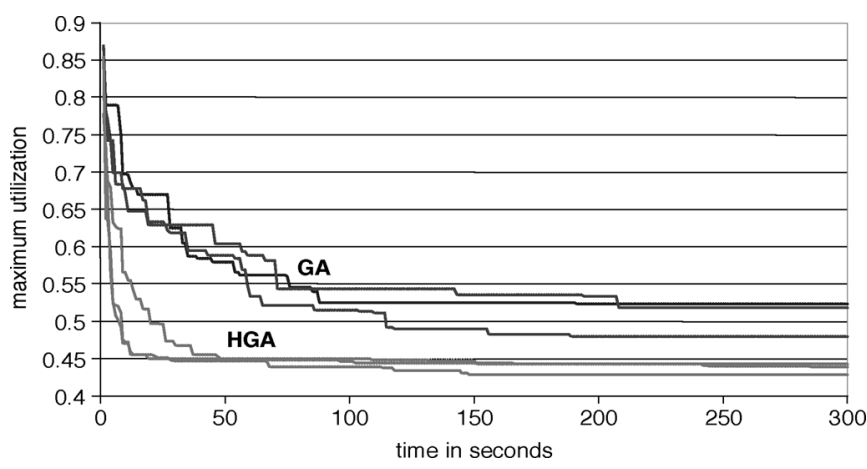

Fig. 5. Comparison of HGA and GA.

heuristic ends. Otherwise, the procedure is repeated for the link, which now has the highest utilization.

For routing protocols with delay and $i \mathrm{~cm}$ metrics there are several possibilities of diverting traffic from an individual link. We can increase the delay weight as well as the $i \mathrm{~cm}$ weight. Additionally, increasing the delay weight while at the same time decreasing the $i \mathrm{~cm}$ weight (or vice versa) could also deflect some of the traffic and, thus, lead to the reduction of a link's load. In our implementation, all four weight modification possibilities are taken into account. However, preference is given to the increase of the delay component and weight changes are checked for their effectiveness in the following order:

1) increment delay weight;

2) increment $i \mathrm{~cm}$ weight;

3) increment delay weight and decrement $i \mathrm{~cm}$ weight;

4) decrement delay weight and increment $i \mathrm{~cm}$ weight.

Weight modifications are carried out repeatedly as long as they do not lead to an increase of the maximum link utilization in the network. The heuristic ends once a local minimum is reached.

In all cases, it is guaranteed that the run time of the local search process is bounded. For single-metric routing, this is obvious, since the algorithm only increments link weights and, therefore, is bounded by the maximum metric threshold $M_{\max }$. For the dual-metric case, guaranteeing a bounded run time is somewhat trickier. As long as we only increase metric values, we do not run into problems. The heuristic will eventually halt when it reaches the maximum values. However, since two of the four metric modification options decrease one of the link metric values, we have to assure that the algorithm does not get caught in an infinite loop. Therefore, our program records the states that have been assumed during the search process. If the heuristic returns to an already visited state, the search stops. This way, a limited run time is again guaranteed.

The benefits of the local search heuristic are demonstrated in the graph of Fig. 5. The graph shows the typical development of the best maximum utilization value per generation over time for three runs of the pure genetic algorithm (GA) and three runs of the hybrid version (HGA). In the given example, the total computation time is 300 seconds on a Linux PC with Pentium 4 processor. For the GA, the population size is 120 , while for the HGA, a size of 20 is sufficient. After 300 seconds, the GA has produced about 600 generations, i.e., it has evaluated around 72000 individual strings. During the same time, the HGA has 
TABLE III

COMPLEXITY AND SOLUTION TIMES FOR THE MIP APPROACH

\begin{tabular}{|c|c|c|c|c|c|c|c|c|c|c|}
\hline & & & \multicolumn{5}{|c|}{ OSPF } & \multicolumn{4}{|c|}{ EIGRP } \\
\hline$|\mathcal{V}|$ & $|\mathcal{E}|$ & $|\mathcal{F}|$ & rows & cols & non-zeros & $t$ & rows & cols & non-zeros & $t$ \\
\hline 5 & 12 & 20 & 306 & 114 & 1146 & $<1 \mathrm{~s}$ & 1406 & 644 & 4486 & $7 \mathrm{~s}$ \\
\hline 6 & 18 & 30 & 600 & 187 & 2345 & $<1 \mathrm{~s}$ & 2017 & 824 & 6764 & $116 \mathrm{~s}$ \\
\hline 7 & 20 & 42 & 923 & 275 & 3700 & $193 \mathrm{~s}$ & 2853 & 1149 & 9650 & $213 \mathrm{~s}$ \\
\hline 8 & 22 & 32 & 1126 & 363 & 4480 & $285 \mathrm{~s}$ & 3601 & 1509 & 11968 & - \\
\hline
\end{tabular}

created 900 generations with a total number of 18000 strings. It is noteworthy that although the population size of the HGA is only a third of the GA's population size, it returns better solutions from the beginning. Therefore, the results, which are presented in the remainder of this paper, were produced with the hybrid genetic algorithm.

\section{NUMERICAL RESUlTS AND DISCUSSION}

In this section, the applicability of the optimization procedure is demonstrated and results for various sample networks are discussed.

\section{A. Applicability of the MIP Model}

The mixed integer programming (MIP) model can be validated for small networks using CPLEX. At this point, we are not interested in the actual results of the optimization program, i.e., the final maximum link utilization value in the network. Rather, we would like to demonstrate the applicability of the models and point out the difference in complexity of the optimization problem when using single-metric and dual-metric protocols. Table III summarizes the complexity for small scenarios with 5 to 8 nodes, 12 to 22 edges, and 20 to 32 traffic flows. The table lists the number of rows and columns of the optimization system, as well as the number of non-zero elements in the matrix. The solution times are given in seconds. As we can see, the OSPF model, which is a reduced form of the model given in Section IV, can be solved optimally within a short amount of time (in some scenarios $t<1$ second), while the complexity and the solution times of the EIGRP model increase quickly. For the network with 8 nodes and 22 links, the solver does not return an EIGRP solution within a reasonable amount of time. The sample scenarios help validate the routing optimization model. However, they also expose its computational complexity, making it intractable for larger network instances.

\section{B. Comparison of OSPF and EIGRP Optimization}

In order to compare routing optimization with OSPF and EIGRP, the hybrid genetic algorithm of the previous section is utilized and results for various sample networks are presented. Table IV provides a summary of the network scenarios, specifying the number of nodes, edges, and flows. The network topologies are illustrated in Fig. 6. All links are bidirectional and have the same capacity. The traffic flows were generated randomly.

Network N11 is a slightly modified version of the network specified by the COST 239 project [28] and network $N 20$ was published in [29]. Both networks, N11 and N2O, are flat networks without hierarchical structure. Scenario N40 represents a network with two hierarchy levels. Each access node is connected to two backbone nodes. Network N5O is a hierarchical
TABLE IV

NETWORK SCENARIOS

\begin{tabular}{|l|c|c|c|c|c|}
\cline { 2 - 6 } \multicolumn{1}{c|}{} & N11 & N20 & N40 & N50 & N100 \\
\hline \# nodes $|\mathcal{V}|$ & 11 & 20 & 40 & 50 & 100 \\
\hline \# links $|\mathcal{E}|$ & 48 & 102 & 150 & 126 & 330 \\
\hline \# flows $|\mathcal{F}|$ & 110 & 380 & 500 & 2450 & 9900 \\
\hline
\end{tabular}
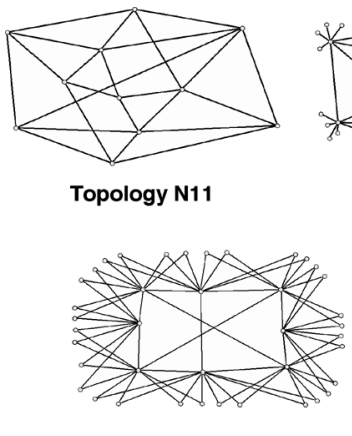

Topology $\mathbf{N} 40$
Topology N11

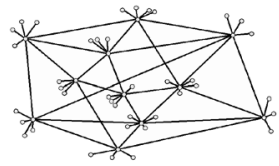

Topology N50

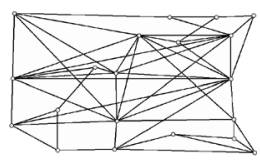

Topology N20
Fig. 6. Network topologies.

version of N11 where each backbone router connects three or four access nodes. Finally, scenario N1OO represents a network where several access clusters are connected through a rather sparse backbone network. Furthermore, direct connections between neighboring access clusters exist.

Table V summarizes the routing optimization results for the five selected network scenarios. In order to better interpret the results, we compare the outcome of each optimization run with the characteristics of "hop-based shortest-path routing". With this we denote the routing solution, which arises if all links are configured with equal weights for the delay metric as well as the $i \mathrm{~cm}$ metric. Note that in this case OSPF and EIGRP produce the same routes. Furthermore, a lower bound, which is based on the reduced linear program, is given.

For each network scenario the optimized maximum utilization value $\rho_{\max }$ as well as the computation time $t$ are listed. Since genetic algorithms do not guarantee to find the optimum, the computations were carried out several times for each scenario with various parameter settings. The table contains the best solution for each scenario and the corresponding computation time.

Additionally, the number of modified link weights $n_{\text {metric }}$ and the number of affected traffic flows $n_{\text {rerouted }}$ are given. Here, we assume that the network was initially configured to implement hop-based shortest-path routing where each link weight was set to half the maximum permissible metric value (in our case, $0.5 \cdot W_{\max }=10$ ). Thus, a higher value for $n_{\text {metric }}$ means that a greater configuration effort is required in order to achieve optimized routing. Similarly, larger values 
TABLE V

RESULTS OF OSPF/EIGRP ROUTING OPTIMIZATION (BEST RESULTS IN BOLD)

\begin{tabular}{|c|c|c|c|c|c|c|}
\hline & & N11 & $\mathbf{N 2 0}$ & N40 & N50 & N100 \\
\hline \multicolumn{7}{|c|}{ Hop-based Shortest-Path Routing } \\
\hline Single Path & $\rho_{\max }$ & 1.0446 & 1.0966 & 0.8692 & 1.0446 & 1.3087 \\
\hline ECMP & $\rho_{\max }$ & 0.7608 & 0.7911 & 0.8819 & 0.7608 & 1.1626 \\
\hline \multicolumn{7}{|c|}{ Optimized Routing } \\
\hline Lower Bound & $\rho_{\max }$ & 0.5476 & 0.4364 & 0.2596 & 0.5476 & 0.57 \\
\hline \multirow[t]{4}{*}{ OSPF } & $\rho_{\max }$ & 0.6079 & 0.4604 & 0.4240 & 0.6079 & 0.6730 \\
\hline & $n_{\text {metric }}$ & $43(90 \%)$ & $95(93 \%)$ & $101(67 \%)$ & $43(34 \%)$ & $290(88 \%)$ \\
\hline & $n_{\text {rerouted }}$ & $32(29 \%)$ & $159(42 \%)$ & $113(23 \%)$ & $657(27 \%)$ & $5631(57 \%)$ \\
\hline & $t$ & $225 \mathrm{~s}$ & $259 \mathrm{~s}$ & $632 \mathrm{~s}$ & $225 \mathrm{~s}$ & $29.7 \mathrm{~min}$ \\
\hline \multirow{4}{*}{$\begin{array}{l}\text { OSPF } \\
\text { ECMP }\end{array}$} & $\rho_{\max }$ & 0.5702 & 0.4583 & 0.4060 & 0.5702 & 0.6405 \\
\hline & $n_{\text {metric }}$ & $38(79 \%)$ & $92(90 \%)$ & $78(52 \%)$ & $38(30 \%)$ & $311(94 \%)$ \\
\hline & $n_{\text {rerouted }}$ & $33(30 \%)$ & $232(61 \%)$ & $217(43 \%)$ & $695(28 \%)$ & $7096(72 \%)$ \\
\hline & $t$ & $12.7 \mathrm{~min}$ & $10.5 \mathrm{~min}$ & $574 \mathrm{~s}$ & $12.7 \mathrm{~min}$ & $3.6 \mathrm{~h}$ \\
\hline \multirow[t]{4}{*}{ EIGRP } & $\rho_{\max }$ & 0.5934 & 0.4476 & 0.3617 & 0.5576 & 0.6582 \\
\hline & $n_{\text {metric }}$ & $92(96 \%)$ & $194(95 \%)$ & $282(94 \%)$ & $214(85 \%)$ & $631(96 \%)$ \\
\hline & $n_{\text {rerouted }}$ & $33(30 \%)$ & $170(45 \%)$ & $150(30 \%)$ & $550(22 \%)$ & $5963(60 \%)$ \\
\hline & $t$ & $368 \mathrm{~s}$ & $334 \mathrm{~s}$ & $315 \mathrm{~s}$ & $169 \mathrm{~s}$ & $1.2 \mathrm{~h}$ \\
\hline \multirow{4}{*}{$\begin{array}{l}\text { EIGRP } \\
\text { ECMP }\end{array}$} & $\rho_{\max }$ & 0.5678 & 0.4406 & 0.3617 & 0.5574 & 0.6405 \\
\hline & $n_{\text {metric }}$ & $92(96 \%)$ & $193(95 \%)$ & $282(94 \%)$ & $150(60 \%)$ & $311(47 \%)$ \\
\hline & $n_{\text {rerouted }}$ & $32(29 \%)$ & $220(58 \%)$ & $203(41 \%)$ & $657(27 \%)$ & $7096(72 \%)$ \\
\hline & $t$ & $180 \mathrm{~s}$ & $424 \mathrm{~s}$ & $315 \mathrm{~s}$ & $19.8 \mathrm{~min}$ & $3.6 \mathrm{~h}$ \\
\hline
\end{tabular}

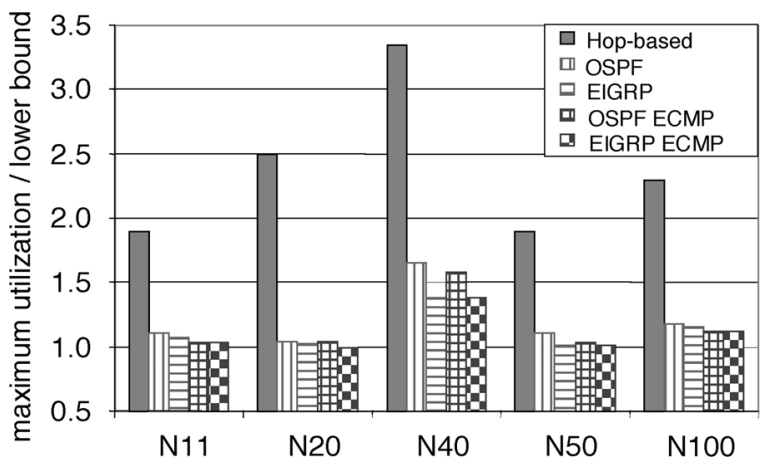

Fig. 7. Comparison of optimization results for OSPF and EIGRP protocols.

for $n_{\text {rerouted }}$ indicate that more flows need to be diverted from their hop-based shortest path for the sake of routing optimization. This number could be used as a measure for the expected service degradation experienced during the transition period when switching from the standard routing configuration to the optimized one.

Table V clearly shows that in all cases routing optimization can drastically reduce the maximum link utilization, often achieving a utilization value that comes close to the lower bound. As expected, EIGRP is able to achieve lower utilization values than OSPF, and load sharing (although it is quite limited for destination-based routing protocols) often allows further QoS enhancement. However, in order to achieve the optimized utilization values it is usually required to modify a rather high portion of link weights. In some cases, over $90 \%$ of the link metric values have to be changed, affecting up to $60 \%$ of all flows (e.g., N20, OSPF ECMP).

Fig. 7 illustrates the results of routing optimization based on OSPF and EIGRP with and without equal-cost multipath option. In the graph, the utilization is given as a factor of the lower bound. Higher values correspond to lower network QoS, while a value of 1 indicates that the lower bound is reached. To illustrate

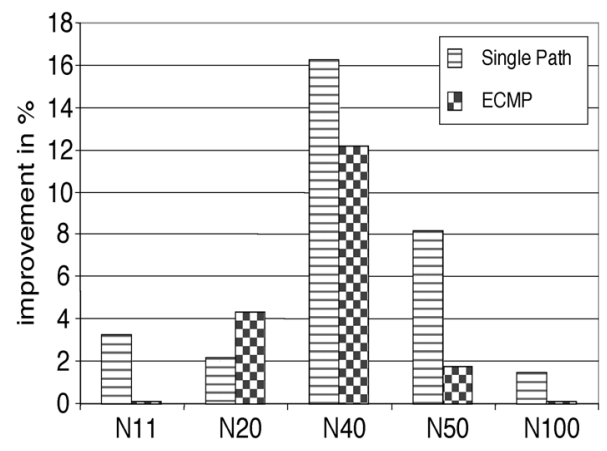

Fig. 8. Relative improvement of EIGRP over OSPF.

the impact of routing optimization, the maximum utilization of the original configuration with hop-based single-shortest-path routing is shown. For the sake of clarity, the value for hop-based ECMP routing is omitted.

Fig. 8 demonstrates the increased optimization potential of dual-metric routing. The graph depicts the improvement of network QoS (i.e., the additional utilization reduction), which can be achieved by EIGRP optimization over OSPF optimization. The differences between the two approaches are given as fractions of the OSPF results, i.e., $\left(\rho_{\max }(O S P F)-\rho_{\max }(E I G R P)\right) / \rho_{\max }(O S P F)$. In some scenarios, the difference is negligibly small (below $2 \%$ in $N 50$, ECMP and N100, Single Path) or even zero (N11, ECMP and N100 ECMP). For other topologies, the improvement can be striking (e.g., $16 \%$ in N40, Single Path). EIGRP optimization performs especially well in networks with hierarchical structures such as N4O and N5O. This observation is consistent with the discussion of routing optimization principles earlier in this paper. In these scenarios, EIGRP can play out its advantage of being able to differentiate routes with common intermediate nodes by utilizing the $i \mathrm{~cm}$ link weights. However, it is also interesting to note that for these cases the configuration effort 
(i.e., the percentage of modified link weights) is significantly higher for EIGRP-based optimization than for the respective OSPF-based counterpart (e.g., $94 \%$ versus $52 \%$ for $N 40$, ECMP). In scenarios with comparable optimization results for EIGRP and OSPF, the proportion of modified link weights are similar.

\section{SUMMARY}

This paper deals with traffic engineering based on link weight optimization, which is known to provide an appropriate means for increasing QoS in networks with unevenly distributed traffic and localized overload situations. While previous work has focused on routing protocols with additive link metrics only (shortest-path routing), we propose to extend this method and utilize routing protocols that consider multiple metric types. Specifically, our approach makes use of protocols that include additive delay weights as well as concave bandwidth weights in their route computation procedure. It can be shown that the consideration of these two metric types benefits routing optimization as it increases the flexibility of the route adjustment process.

In a first step, the link weight optimization problem is formulated as a mixed-integer linear program with link delay and capacity weights as the principle optimization variables and the minimization of the maximum link utilization as the objective. It is our intention to accurately capture the specifics of the underlying dual-metric route computation scheme. As a consequence, the mixed-integer program is rather complex, requiring a large number of variables and constraints. Although optimal solutions can only be found for small networks, we believe that the mixed-integer program provides a valuable contribution by giving an accurate description of the problem at hand.

In order to demonstrate the benefits of dual-metric based routing optimization for larger networks, we present a hybrid genetic algorithm, which incorporates the specific properties of the underlying routing protocol. This algorithm extends the pure genetic framework by employing a local search heuristic within its evaluation stage. This heuristic improves the quality and speed of the optimization process significantly. On the basis of several network scenarios, the capability of routing optimization with OSPF (one metric) and EIGRP (two metrics) is demonstrated. In all cases, optimizing the link weights reduces the maximum link utilization substantially as compared with hop-based shortest-path routing. For some scenarios, the final results are very close to the theoretical lower bound, which is computed by solving a modified version of the linear multicommodity flow problem.

As for the advantage of dual-metric routing optimization, it can be shown that it is indeed able to outperform its single-metric counterpart. Especially in network scenarios with hierarchical topology structure, it has the potential to make finer adjustments to the route pattern and, thus, achieve greater QoS increase. In two of the considered scenarios, the additional reduction of the maximum link utilization is significant, corresponding to gains of up to $16 \%$. However, one has to be aware of the higher configuration effort of dual-metric routing as it requires more weights to be adjusted. Thus, we have a tradeoff between quality gain and operational complexity. Furthermore, it should be noted that it is difficult to predict the final gain of the two optimization procedures without actually performing the optimization. In practice, it is therefore recommended to carry out the optimization procedures for both options and then choose the one that is more adequate.

In conclusion, dual-metric routing optimization is a promising tool for traffic engineering based on today's interior routing protocols. Since many routers that are employed in networks around the world could be configured to support additive as well as concave link metrics, dual-metric routing optimization should be given consideration as a means for traffic engineering before switching to more advanced technologies.

\section{REFERENCES}

[1] D. Awduche, A. Chiu, A. Elwalid, I. Widjaja, and X. Xiao, "Overview and principles of Internet traffic engineering," IETF, RFC 3272, May 2002.

[2] J. Moy, “OSPF version 2," IETF, RFC 2328, Apr. 1998.

[3] A. Zinin, Cisco IP Routing. Boston, MA: Addison-Wesley, 2002.

[4] Z. Wang and J. Crowcroft, "Analysis of shortest-path routing algorithms in a dynamic network environment," Comput. Commun. Rev., vol. 22, no. 2, pp. 63-71, 1992.

[5] E. Rosen, A. Viswanathan, and R. Callon, "Multiprotocol label switching architecture," IETF, RFC 3031, Jan. 2001.

[6] D. Awduche, J. Malcolm, J. Agogbua, M. O’Dell, and J. McManus, "Requirements for traffic engineering over MPLS," IETF, RFC 2702, Sep. 1999.

[7] A. Bley, M. Grötschel, and R. Wessäly, "Design of broadband virtual private networks: model and heuristics for the B-WiN," Konrad-ZuseZentrum für Informationstechnik, Berlin, Germany, Preprint SC 98-13, Mar. 1998.

[8] B. Fortz and M. Thorup, "Internet traffic engineering by optimizing OSPF weights," in Proc. IEEE INFOCOM 2000, pp. 519-528.

[9] B. Fortz, J. Rexford, and M. Thorup, "Traffic engineering with traditional IP routing protocols," IEEE Commun. Mag., vol. 40, no. 10, pp 118-124, Oct. 2002.

[10] B. Fortz and M. Thorup, "Optimizing OSPF/IS-IS weights in a changing world," IEEE J. Sel. Areas Commun., vol. 20, no. 5, pp. 756-766, May 2002.

[11] M. Ericsson, M. Resende, and P. Pardalos, "A genetic algorithm for the weight setting problem in OSPF routing," J. Combinat. Optim., vol. 6, pp. 299-333, 2002.

[12] A. Riedl, "A versatile genetic algorithm for network planning," in Proc. Open European Summer School on Network Management and Operation (EUNICE'98), Munich, Germany, 1998, pp. 97-103.

[13] M. Resende, "A memetic algorithm for OSPF routing," presented at the 6th INFORMS Telecom Conf., Boca Raton, FL, Mar. 2002.

[14] K. Ramakrishnan and M. Rodrigues, "Optimal routing in shortest-path data networks,” Bell Labs Tech. J., pp. 117-138, Jan.-Jun. 2001.

[15] R. Ahuja, T. Magnanti, and J. Orlin, Network Flows-Theory, Algorithms, and Applications. Upper Saddle River, NJ: Prentice Hall, 1993.

[16] Y. Wang, Z. Wang, and L. Zhang, "Internet traffic engineering without full mesh overlaying," in Proc. IEEE INFOCOM 2001, pp. 565-571.

[17] A. Sridharan, R. Guérin, and C. Diot, "Achieving near-optimal traffic engineering solutions for current OSPF/IS-IS networks," in Proc. IEEE INFOCOM 2003, pp. 1167-1177.

[18] D. Staehle, S. Köhler, and U. Kohlhaas, "Towards an optimization of the routing parameters for IP networks," Univ. Würzburg, Germany, Tech. Rep. 258, May 2000.

[19] S. Köhler, D. Staehle, and U. Kohlhaas, "Optimization of IP routing by link cost specification," presented at the Internet Traffic Engineering and Traffic Management 15th ITC Specialist Seminar, Würzburg, Germany, Jul. 2002.

[20] CPLEX. ILOG Inc. [Online]. Available: http://www.ilog.com

[21] J. Milbrandt, S. Köhler, D. Staehle, and L. Berry, "Decomposition of large IP networks for routing optimization," Univ. Würzburg, Tech. Rep. 293, Feb. 2002.

[22] E. Mulyana and U. Killat, "An alternative genetic algorithm to optimize OSPF weights," presented at the Internet Traffic Engineering and Traffic Management, 15th ITC Specialist Seminar, Würzburg, Germany, Jul. 2002. 
[23] E. Mulyana and U. Killat, "A hybrid genetic algorithm approach for OSPF weight setting problem," presented at the 2nd Polish-German Teletraffic Symp. (PGTS 2002), Gdansk, Poland, Sep. 2002.

[24] K. Holmberg and D. Yuan, "Optimization of Internet protocol network design and routing," Dept. Math., Linköping Inst. Technol., Linköping, Sweden, Research Report LiTH-MAT-R-2001-07, Mar. 2001.

[25] K. Holmberg and D. Yuan, "Optimal network design and routing for IP traffic," presented at the 3rd Int. Workshop on Design of Reliable Communication Networks (DRCN 2001), Budapest, Hungary, Oct. 2001.

[26] A. Riedl, "A hybrid genetic algorithm for routing optimization in IP networks utilizing bandwidth and delay metrics," presented at the IEEE Workshop on IP Operations and Management (IPOM), Dallas, TX, Oct. 2002.

[27] D. Goldberg, Genetic Algorithms in Search, Optimization and Machine Learning. Reading, MA: Addison-Wesley, 1989.

[28] COST 239: Ultra-High Capacity Optical Transmission Networks. 1998 [Online]. Available: http://www.barolo.ita.hsr.ch/cost239

[29] S. Köhler and A. Binzenhöfer, "MPLS traffic engineering in OSPF networks—a combined approach," Univ. Würzburg, Germany, Tech. Rep. 304, Feb. 2003.

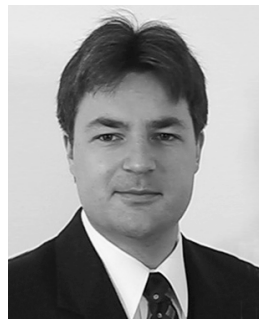

Anton Riedl (M'99) studied electrical engineering at Munich University of Technology (TUM), Germany, and, as a Fulbright Scholar, at Purdue University, West Lafayette, IN. He received the M.S. degree in electrical engineering from Purdue University in 1995 and the Dipl.-Ing. degree from TUM in 1996. From 1997 to 2003, he was a member of the research and teaching staff at the Institute of Communication Networks at TUM. He received the Dr.-Ing. degree in electrical engineering from TUM in 2004.

Since 2003, he has been an Assistant Professor in the Department of Physics, Computer Science and Engineering at Christopher Newport University, Newport News, VA. His research interests are in the area of multiservice IP networking (including architectural and protocol aspects as well as network planning and optimization issues), wireless sensor networking, sensor webs, and network security.

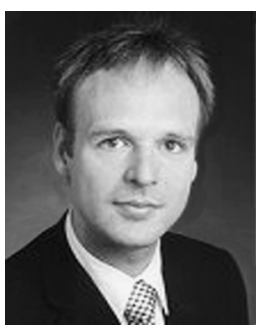

Dominic A. Schupke (M'97) received the Dipl.-Ing. degree from RWTH Aachen, Germany, in 1998, and the Dr.-Ing. degree from Munich University of Technology (TUM), Germany, in 2004.

In February 2004, he joined Siemens, Corporate Technology, Munich, Germany, where he became a Senior Research Scientist. In October 2006, he moved to Siemens Networks, which in April 2007 became part of the joint venture Nokia Siemens Networks (NSN). His research interests are in the area of network architectures and protocols (optical, IP/MPLS, Ethernet, multilayer networks), routing and wavelength assignment, recovery methods, availability analysis, network optimization, and network planning. 